\title{
TWO CONTACT PROBLEMS IN ANISOTROPIC ELASTICITY
}

\author{
D. L. CLEMENTS \\ (Received 8 May 1970; revised 21 September 1970) \\ Communicated by A. F. Pillow
}

In this paper we consider two contact problems for an anisotropic elastic half-space in which the stress is independent of one of the Cartcsian co-ordinates. The results hold for the most general anisotropy in which no symmetry elements of the material are assumed. Problems of this type have been considered by Brilla [1], Clements [2], Galin [3], Green and Zerna [4] and Milne-Thomson [5], but the work of these authors is only applicable to a restricted class of anisotropic materials. We begin in section 1 by deriving some fundamental equations for the stress and displacement. It will be noted that at an early stage (equation (7)) the roots of a sextic polynomial are required. Since these cannot be obtained explicitly any application of the general theory must, of necessity, be numerical. However the calculation of the stress and displacement is simplified if certain symmetry elements of the material are assumed and the way in which these simplifications occur is indicated in section 2. In sections 3 and 4 we consider contact problems in which the elastic half-space is indented by a rigid body. For the problem considered in section 3 , the rigid body is assumed to be able to move relative to the surface of the half-space, while for the problem considered in section 4 it is assumed to be linked to the half-space.

\section{General equations}

The stresses $\sigma_{i j}$ are related to the elastic displacement $u_{k}$ by the equations

$$
\sigma_{i j}=c_{l j k l} \frac{\partial u_{k}}{\partial x_{l}}
$$

where $i, j, k, l=1,2,3$ and the convention of summing over a repeated Latin suffix is used. The elastic moduli $c_{i j k l}$ have the symmetry properties

$$
c_{i j k l}=c_{j i k l}=c_{i j l k}=c_{k l i j} .
$$

On substituting (1) in the equilibrium equations 
we obtain

$$
\frac{\partial \sigma_{i j}}{\partial x_{j}}=0,
$$

$$
c_{i j k l} \frac{\partial^{2} u_{k}}{\partial x_{j} \partial x_{l}}=0 .
$$

Now we suppose that $u_{k}$ is independent of $x_{3}$ and take

$$
u_{k}=A_{k} f\left(x_{1}+p x_{2}\right) \text {. }
$$

where $f(z)$ is an analytic function of the complex variable $z ;(5)$ is a solution of the equations (4) provided the constant vector $A_{k}$ satisfies the equations

$$
\left(c_{i 1 k 1}+p c_{i 1 k 2}+p c_{i 2 k 1}+p^{2} c_{i 2 k 2}\right) A_{k}=0 .
$$

Values of $A_{k}$, not identically zero, can be found to satisfy these equations if $p$ is a root of the sextic equation

$$
\left|c_{i 1 k 1}+p c_{i 1 k 2}+p c_{i 2 k 1}+p^{2} c_{i 2 k 2}\right|=0 \text {. }
$$

By applying the condition that the strain energy density should be positive for any state of strain Eshelby. Read and Shockley [6] have proved that equation (7) has no real root, so that the roots occur in complex conjugate pairs. The three roots with positive imaginary part will be denoted by $p_{\alpha}(\alpha=1,2,3)$ with complex conjugates $\bar{p}_{\alpha}$; the corresponding values of $A_{\alpha}$ obtained from equation (6) are $A_{k \alpha}$ and $\bar{A}_{k \alpha}$. Summation over $\alpha$, and generally over Greek suffices, will always be indicated explicitly. It will be assumed that the roots $p_{\alpha}$ are all distinct; equal roots being regarded as the limiting case of distinct roots. A general expression for the displacement may then be written

$$
u_{k}=\sum_{\alpha} A_{k \alpha} f_{\alpha}\left(z_{\alpha}\right)+\sum_{\alpha} \bar{A}_{k \alpha} \bar{f}_{\alpha}\left(\bar{z}_{\alpha}\right)
$$

where $z_{\alpha}=x_{1}+p_{a} x_{2}$. From (1) we write the stresses as

$$
\sigma_{i j}=\sum_{\alpha} L_{i j \alpha} f_{\alpha}^{\prime}\left(z_{\alpha}\right)+\sum_{\alpha} \bar{L}_{i j \alpha} \bar{f}_{\alpha}^{\prime}\left(\bar{z}_{\alpha}\right)
$$

where

$$
L_{i j \alpha}=\left(c_{i j k 1}+p_{\alpha} c_{i j k 2}\right) A_{k \alpha}
$$

and primes denote differentiation with respect to $z_{\alpha}$. It will be useful subsequently to have an alternative form of the equations (9) and (10). We define

$$
\sum_{\alpha} L_{i 2 \alpha} f_{\alpha}(z)=\phi_{i}(z)
$$

where the $\phi_{i}(z)(i=1,2,3)$ are analytic functions of the complex variable $z$. Stroh [7] has shown that the matrix $\left[L_{i 2 \alpha}\right]$ is non-singular so we may write 


$$
f_{\alpha}(z)=M_{\alpha j} \phi_{j}(z),
$$

where

$$
\sum_{\alpha} L_{i 2 \alpha} M_{\alpha j}=\delta_{i j}
$$

Hence substituting (12) in (8) and (9) it follows that

$$
\begin{aligned}
& u_{k}=\sum_{\alpha} A_{k \alpha} M_{\alpha j} \phi_{j}\left(z_{\alpha}\right)+\sum_{\alpha} \bar{A}_{k \alpha} \bar{M}_{\alpha j} \bar{\phi}_{j}\left(\bar{z}_{\alpha}\right), \\
& \sigma_{i j}=\sum_{\alpha}^{\alpha} L_{i j \alpha} M_{\alpha k} \phi_{k}^{\prime}\left(z_{\alpha}\right)+\sum_{\alpha} \bar{L}_{i j \alpha} \bar{M}_{\alpha k} \bar{\phi}_{k}^{\prime}\left(\bar{z}_{\alpha}\right) .
\end{aligned}
$$

In the subsequent sections, we shall be concerned with contact problems for an elastic material occupying the lower half-space $x_{2}<0$. Hence for such problems the $\phi_{i}(z)$ occurring in (14) and (15) are defined and analytic in the region $x_{2}<0$, but in the region $x_{2}>0$ where the material is absent these functions are undefined. We can therefore define the $\phi_{i}(z)$ for $x_{2}>0$ in any way we please. It will prove useful to define

$$
\phi_{i}(z)=-\overline{\phi_{i}(\bar{z})}, \quad \phi_{i}^{\prime}(z)=-\overline{\phi_{i}^{\prime}(\bar{z})}, \quad x_{2}>0,
$$

and hence

$$
\phi_{i}(\bar{z})=-\overline{\phi_{i}(z)}, \phi_{i}^{\prime}(\bar{z})=-\overline{\phi_{i}^{\prime}(z)}, \quad x_{2}<0 .
$$

Hence (14) and (15) may be written

$$
\begin{array}{lll}
u_{k}=\sum_{\alpha} A_{k \alpha} M_{\alpha j} \phi_{j}\left(z_{\alpha}\right)-\sum_{\alpha} \bar{A}_{k \alpha} \bar{M}_{\alpha j} \phi_{j}\left(\bar{z}_{\alpha}\right), & x_{2}<0, \\
\sigma_{i j}=\sum_{\alpha} L_{i j \alpha} M_{\alpha k} \phi_{k}^{\prime}\left(z_{\alpha}\right)-\sum_{\alpha} \tilde{L}_{i j \alpha} \bar{M}_{\alpha k} \phi_{k}^{\prime}\left(\bar{z}_{\alpha}\right), & x_{2}<0 .
\end{array}
$$

On the plane $x_{2}=0(18)$ and (19) reduce to

$$
\begin{aligned}
u_{k} & =B_{k j} \phi_{j}^{-}\left(x_{1}\right)-\bar{B}_{k j} \phi_{j}^{+}\left(x_{1}\right), \\
\sigma_{i 2} & =\phi_{i}^{\prime-}\left(x_{1}\right)-\phi_{i}^{\prime+}\left(x_{1}\right),
\end{aligned}
$$

where

and

$$
\lim _{x_{2} \rightarrow 0+} \phi_{i}(z)=\phi_{i}^{+}\left(x_{1}\right), \lim _{x_{2} \rightarrow 0-} \phi_{i}(z)=\phi_{i}^{-}\left(x_{1}\right)
$$

$$
B_{k j}=\sum_{\alpha} A_{k \alpha} M_{\alpha j} .
$$

Finally, in this section, if we require the $\phi_{i}^{\prime}(z)$ to be analytic in the whole plane cut along the real axis from $x_{1}=-b$ to $x_{1}=a$, where $a$ and $b$ are positive constants, then it follows from (21) that on the plane $x_{2}=0$

$$
\sigma_{i 2}=0, \quad x_{1}<-b, x_{1}>a .
$$




\section{Particular cases of elastic symmetry}

The expressions for the stress and displacement derived in the previous section involve the roots of the sextic (7) and in the general case these must, of necessity, be obtained numerically. However there is some simplification if the material exhibits elastic symmetry with respect to one or more of the planes $x_{i}=0(i=1,2,3)$. In the case of elastic symmetry with respect to the $x_{1}=0$ plane, the elastic constants $c_{i j k l}$ with an uneven number of ones occurring in their subscripts are zero, so that the sextic in $p(7)$ reduces to a cubic in $p^{2}$. It is then possible to use equations (6) and (10) to obtain conditions which the $A_{k \alpha}$ and $L_{i j \alpha}$ may be required to satisfy when elastic symmetry of this type exists. Details of the way in which these conditions may be obtained are available in Clements [2]. A similar simplification occurs if there is elastic symmetry with respect to the $x_{2}=0$ plane. For elastic symmetry with respect to the $x_{3}=0$ plane, the elastic constants with an uneven number of threes occurring in their subscripts are zero, so that the sextic (7) factorizes into a quartic and a quadratic. The solution of particular problems for this class of materials has attracted the attention of a number of authors and the contact problems considered in this paper have been solved by Galin [3] for such materials.

\section{Contact problem with friction}

Consider an anisotropic elastic material which occupies the half-space $x_{2}<0$ and suppose the boundary $x_{2}=0$ of the half-space is indented by a rigid punch over the region $-b<x_{1}<a,-\infty<x_{3}<\infty$ where $a$ and $b$ are positive constants. For simplicity we restrict our attention to a single punch although the work may be easily generalised to include the case of several punches. We shall assume that the punch is in a state of limiting equilibrium so that, in either or both of the $x_{1}$ and $x_{3}$ directions, it is subjected to the action of a force equal to the product of the coefficient of friction and the force pressing down the punch. On the plane $x_{2}=0$ we have the following boundary conditions

with

$$
\begin{gathered}
\sigma_{12}=\sigma_{22}=\sigma_{23}=0, \quad x_{1}<-b, x_{1}>a,-\infty<x_{3}<\infty, \\
u_{2}=f\left(x_{1}\right), \sigma_{12}=\mu_{1} \sigma_{22}, \quad \sigma_{23}=\mu_{2} \sigma_{22}, \\
-b<x_{1}<a,-\infty<x_{3}<\infty,
\end{gathered}
$$

$$
\int_{-b}^{a}\left[\sigma_{22}\right]_{\lambda_{2}=0} d x_{1}=-P
$$

where $\mu_{1}$ and $\mu_{2}$ are equal to plus or minus the coefficient of friction and $P$ is the total applied force per unit length. In the absence of friction in the $x_{1}$ or $x_{3}$ 
directions we may put $\mu_{1}$ or $\mu_{2}$ respectively equal to zero. The boundary conditions (24) and (25) will be satisfied if we put

$$
\phi_{1}(z)=\mu_{1} \phi_{2}(z), \quad \phi_{3}(z)=\mu_{2} \phi_{2}(z)
$$

and require the $\phi_{2}^{\prime}(z)$ to be analytic in the whole plane cut along $(-b, a)$ and to be such that

$$
\bar{N}_{2} \phi_{2}^{\prime+}\left(x_{1}\right)-N_{2} \phi_{2}^{\prime-}\left(x_{1}\right)=-f^{\prime}\left(x_{1}\right), \quad-b<x_{1}<a,
$$

where

$$
N_{2}=\mu_{1} B_{21}+B_{22}+\mu_{2} B_{23} .
$$

The problem (28) is a special case of the Hilbert problem. The appropriate solution is given by (see Muskhelishvili [8])

$$
\phi_{2}^{\prime}(z)=\frac{-X(z)}{2 \pi i \bar{N}_{2}} \int_{-b}^{a} \frac{f^{\prime}(t) d t}{X^{+}(t)(t-z)}+K X(z)
$$

where

$$
\begin{gathered}
X(z)=(z-a)^{\gamma-1}(z+b)^{-\gamma}, \\
\gamma=\frac{1}{2 \pi i} \log \left(N_{2} / \bar{N}_{2}\right),
\end{gathered}
$$

where the argument of $N_{2} / \bar{N}_{2}$ is chosen between 0 and $2 \pi$ and we select the branch of $X(z)$ such that $z X(z) \rightarrow 1$ as $|z| \rightarrow \infty$. In (30), $X^{+}(t)$ refers to the value on $x_{2}=0+$ of the function $X(z)$ with $x_{1}$ replaced by the variable of integration $t$. Also using the condition (26) we may show that the constant $K$ occurring in (30) is given by

$$
K=P / 2 \pi i \text {. }
$$

Equation (30) will only provide a valid solution to the problem of this section if $\bar{N}_{2} \neq 0$. Thus the solution will not hold for real values of $\mu_{1}$ and $\mu_{2}$ which give rise, through (29), to $\bar{N}_{2}=0$. However if such values of $\mu_{1}$ and $\mu_{2}$ exist then we may modify the problem as follows. From (20) and (27) it follows that when $-b<x_{1}<a$

$$
u_{k}\left(x_{1}, 0\right)=N_{k} \phi_{2}^{-}\left(x_{1}\right)-\bar{N}_{k} \phi_{2}^{+}\left(x_{1}\right), \quad k=1,2,3,
$$

where

$$
N_{k}=\mu_{1} B_{k 1}+B_{k 2}+\mu_{2} B_{k 3} .
$$

Now if $N_{2} \neq 0$ then the shape of the end of the punch (which determines the function $f\left(x_{1}\right)$ in (25)) may take a fairly general form but if $N_{2}=0$ then it follows from (33) that $u_{2}\left(x_{1}, 0\right)=0$ for all $x_{1}$ and hence, if contact is to take place over the whole of the region $-b<x_{1}<a$, we must require the punch to be flat-ended 
so that $f\left(x_{1}\right)=0$. Equation (28) with $N_{2}=0$ is then consistent but it does not enable us to determine $\phi_{2}(z)$. However if the form of $u_{1}\left(x_{1}, 0\right)$ or $u_{3}\left(x_{1}, 0\right)$ can be determined for $-b<x_{1}<a$ then the equation (33) with $k=1$ or 2 may be used in place of (28) to determine $\phi_{2}^{\prime}(z)$. It is of interest to note that, using the method developed by Clements [2], it is possible to show that when the $x_{1}=0$ plane is a plane of elastic symmetry $B_{22}$ and $B_{23}$ have zero real part while $B_{21}$ has zero imaginary part. Thus, in this case, real values of $\mu_{1}$ and $\mu_{2}$ which give $N_{2}=0$ are $\mu_{1}=0$ and $\mu_{2}=-B_{22} / B_{23}$. Similarly Clements' method may be used to show that when the $x_{2}=0$ plane is a plane of elastic symmetry $B_{21}$ and $B_{23}$ have zero imaginary part and $B_{2 ?}$ has zero real part. Thus, when such symmetry exists, it is not possible to choose real values of $\mu_{1}$ and $\mu_{2}$ to give $N_{2}=0$ unless $B_{22}=0$.

\section{Contact problem with linkage}

In this case we assume that the punch is rigidly linked to the half-space so that the boundary conditions on the plane $x_{2}=0$ are

$$
\begin{gathered}
\sigma_{12}=\sigma_{22}=\sigma_{23}=0, \quad x_{1}<-b, \quad x_{1}>a, \quad-\infty<x_{3}<\infty, \\
u_{k}=f_{k}\left(x_{1}\right), \quad-b<x_{1}<a, \quad-\infty<x_{3}<\infty,
\end{gathered}
$$

with

$$
\int_{-b}^{a}\left[\sigma_{j 2}\right]_{x_{2}=0} d x_{1}=-P_{j}, \quad j=1,2,3 .
$$

The boundary conditions (34) and (35) will be satisfied if we choose functions $\phi_{i}^{\prime}(z)$ which are analytic in the whole plane cut along $(-b, a)$ and are such that

$$
\bar{B}_{k j} \phi_{j}^{\prime+}\left(x_{1}\right)-B_{k j} \phi_{j}^{\prime-}\left(x_{1}\right)=-f_{k}^{\prime}\left(x_{1}\right), \quad-b<x_{1}<a .
$$

Multiplying by constants $C_{k}$ which are yet to be determined and summing over $k$ it follows that

$$
C_{k} \bar{B}_{k j} \phi_{j}^{\prime+}\left(x_{1}\right)-C_{k} B_{k j} \phi_{J}^{\prime-}\left(x_{1}\right)=-C_{k} f_{k}^{\prime}\left(x_{1}\right), \quad-b<x_{1}<a .
$$

We choose the $C_{k}$ such that

$$
C_{k} \bar{B}_{k j}=D_{j}, \quad C_{k} B_{k j}=\lambda D_{j},
$$

where the $D_{j}$ and $\lambda$ are yet to be determined. Eliminating the $D_{j}$ we obtain

$$
\left(B_{k j}-\lambda \bar{B}_{k j}\right) C_{k}=0 .
$$

These equations have a non-trivial solution if

$$
\left|B_{k j}-\lambda \bar{B}_{k j}\right|=0 \text {, }
$$

which is a cubic in $\lambda$ with roots which we will denote by $\lambda_{\beta}(\beta=1,2,3)$; the cor- 
responding values of $C_{k}$ and $D_{k}$, obtained from (38) and (39), will be denoted by $C_{\beta k}$ and $D_{\beta k}$. Equation (37) may now be written

$$
\left\{D_{\beta j} \phi_{j}^{\prime+}\left(x_{i}\right)\right\}-\lambda_{\beta}\left\{D_{\beta j} \phi_{j}^{\prime-}\left(x_{i}\right)\right\}=-C_{\beta k} f_{k}^{\prime}\left(x_{1}\right), \quad \begin{gathered}
-b<x_{1}<a, \\
\beta=1,2,3 .
\end{gathered}
$$

Equation (41) is a special case of the Hilbert problem. The appropriate solution is given by

where

$$
D_{\beta j} \phi_{j}^{\prime}(z)=\frac{-X_{\beta}(z)}{2 \pi i} \int_{-b}^{a} \frac{C_{\beta k} f_{k}^{\prime}(t) d t}{X_{\beta}^{+}(t)(t-z)}+K_{\beta} X_{\beta}(z), \quad \beta=1,2,3
$$

$$
\begin{aligned}
X_{\beta}(z) & =(z-a)^{\gamma-1}(z+b)^{-\gamma}, \\
\gamma & =\frac{1}{2 \pi i} \log \lambda_{\beta},
\end{aligned}
$$

where we select the branch of $X_{\beta}(z)$ such that $z X_{\beta}(z) \rightarrow 1$ as $|z| \rightarrow \infty$. Also the argument of $\lambda_{\beta}$ is chosen between 0 and $2 \pi$ and the $K_{\beta}$ are constants which may be evaluated by using the conditions (36). Provided the matrix $\left[D_{\beta j}\right]$ is nonsingular we may write

where

$$
\phi_{j}^{\prime}(z)=\sum_{\beta}\left[\frac{-E_{j \beta} X_{\beta}(z)}{2 \pi i} \int_{-b}^{a} \frac{C_{\beta k} f_{k}^{\prime}(t) d t}{X_{\beta}^{+}(t)(t-z)}+E_{j \beta} K_{\beta} X_{\beta}(z)\right]
$$

Equation (19) then gives the stress at all points of the half-space.

\section{References}

[1] J. Brilla, 'Contact problems of an elastic anisotropic half plane' (in Roumanian), Studii si Cercetari Mecan. Apl. Inst. Mecan. Apl., Acad. Rep. Pop. Romine 12, (1961), 959-987.

[2] D. L. Clements, 'The indentation of an anisotropic half space by a rigid punch', J. Aust. Math. Soc. 12 (1971), 75-82.

[3] L. A. Galin, Contact problems in the theory of elasticity (Translation by North Carolina State College Department of Mathematics, 1961), 90-111.

[4] A. E. Green and W. W. Zerna, Theoretical elasticity (O. U. P. 1st edition, 1954), Chapter 9.

[5] L. M. Milne-Thomson, Plane elastic systems (Springer-Verlag, New York 1960), Chapter 7.

[6] J. D. Eshelby, W. T. Read and W. Shockley, 'Anisotropic elasticity with applications to dislocation theory', Acta Met. 1 (1953), 251-259.

[7] A. N. Stroh, 'Dislocations and cracks in anisotropic elasticity', Phil. Mag. 3 (1958), 625-646.

[8] N. I. Muskhelishvili, Some basic problems of the mathematical theory of elasticity, (Translation by J. R. M. Radok, P. Noordhoff, Ltd., Groningen, The Netherlands, 1953).

[9] S. G. Lekhnitskii, Theory of elasticity of an anisotropic elastic body (Holden-Day, 1953).

Department of Theoretical Mechanics

University of Nottingham, England

Present address.

Department of Applied Mathematics

University of Western Ontario, Canada 